\title{
A Journey across the Wall of Varicose Veins: What Physicians Do Not Often See with the Naked Eye
}

\author{
W.M. Renno ${ }^{a}$ F. Saleh ${ }^{a} \quad$ M. Wali ${ }^{b}$ \\ a Department of Human Anatomy, Faculty of Medicine, Kuwait University, Kuwait, Kuwait; \\ ${ }^{b}$ Department of Surgery, College of Medicine, King Khalid University, Abha, Saudi Arabia
}

\section{Key Words}

Varicose veins . Collagen - Elastin $\cdot$ Smooth muscle

\begin{abstract}
Objective: To examine varicose veins (VVs) from inside out in order to help surgeons and general practitioners better understand the pathogenesis of the disease and improve their management. Material and Methods: A comprehensive examination of the wall of $\mathrm{VVs}$ was performed using transmission electron microscopy. The ultrastructural morphology of the collagen, elastin and smooth muscle content of the wall was analyzed in a sample of 10 patients ( 4 male and 6 female) and 10 matched controls aged between 37 and 50 years. $\boldsymbol{R e}$ sults: Analysis of the tunica media revealed that the smooth muscle cells were significantly separated from each other by a marked increase in amorphous and fibrous tissue in which many of the collagen and elastin fibers lost their normal structural arrangement. The cells contained a large number of membrane-bound intracellular vesicles and cytoplasmic vacuoles. The collagen fibers were smaller and thinner than what is commonly seen in normal veins, and they were widely separated from each other. A light electron-lucent center was observed in the middle of the fibers. Similar changes were also seen in the intima and were associated with irregular plaque-like intimal thickening. Conclusion: Our study
\end{abstract}

revealed a significant separation among smooth muscle cells in the wall of VVs, and the presence of an abnormal amorphous extracellular matrix and intracytoplasmic vacuoles could reflect 'unusual' possible secretory and phagocytic roles of smooth muscle cells. This could provide an important explanation for the abnormal contractile function of these cells in VVs.

Copyright (C) 2006 S. Karger AG, Basel

\section{Introduction}

Varicose vein is one of the most common peripheral vascular disorders that both men and women develop at a certain stage during their life. A survey conducted in the UK showed that $10-40 \%$ of people aged between 30 and 70 years develop the disease $[1,2]$. Ten percent of those affected develop complications such as superficial thrombophlebitis, pigmentation, lipodermatosclerosis, hemorrhage, ulceration and increased risk for deep vein thrombosis [3]. Severer chronic venous insufficiency is found in nearly $20 \%$ of working men and women [4], and occupation is ranked high among the predisposing factors [5].

Primary varicose veins (VVs) are characterized by venous backflow and blood stagnation leading to progressive vascular wall remodeling characterized by abnormal dilatation and tortuous appearance [6]. However, the ex-

\section{KARGER}

Fax +4161306 1234 E-Mail karger@karger.ch www.karger.com
(C) 2006 S. Karger AG, Basel

$1011-7571 / 06 / 0151-0009 \$ 23.50 / 0$

Accessible online at:

www.karger.com/mpp
Dr. Waleed Renno

Department of Human Anatomy, Kuwait University, Faculty of Medicine PO Box 24923

Safat 13110 (Kuwait)

Tel. +965 531 2300, ext. 6265, Fax +965 531 9478, E-Mail wrenno@hsc.edu.kw 
act cause of the primary form of varicosis is still poorly understood [7, 8]. Incompetent saphenous vein valves [9, 10], an inherent weakness in the vein wall [3] and the presence of multiple arteriovenous communications [11] are some accepted theories attributed to the pathogenesis of VVs. The common outcome of these theories is an increased pressure in the superficial veins leading to their dilatation and subsequent malfunctioning.

There is a general agreement among investigators of VVs implying that the latter are typically saccular and usually begin peripherally in the tributaries of the long or short saphenous veins but not in the main proximal trunks $[9,12,13]$. Such an agreement also implicates the presence of a strong family history of VVs [14]. However, the nature of the implication of defects in the connective tissue elements and smooth muscle cells (SMCs) in the pathogenesis of VVs remains controversial. Normally, collagen, elastin and smooth muscles control the distensibility of the vein wall. The active tone of the vessel wall is maintained by the tunica media SMCs, which in turn are influenced by autonomic nerves and circulating stimulants. The passive tone is provided by the collagen and elastin fibers. Therefore, a defect in one or more of these components could lead to the loss of tone often seen in VVs [3].

Investigations into the nature of defects of the cellular and extracellular components of the wall of VVs have revealed various results. In a study with experimentally induced hypoxia conducted by Michiels et al. [15], they found an increase in the thickness of venous tunica media associated with extracellular matrix deposition as well as hyperplasia of SMCs resulting from experimentally induced hypoxia and a loss of the vessel tone. The authors concluded that the above alterations in the venous wall were similar to those observed in VVs and, therefore, hypoxia should be investigated in primary varicosis. Light microscopy studies of VVs demonstrated disorganization of the venous wall including varying degrees of intimal thickening, infiltration of fibrous tissue in the tunica intima and media, separation and interruption of the SMCs and fragmentation of elastin fibers [16, 17]. An increase in the collagen content of the wall of VVs associated with a concomitant increase in proteoglycans and a decrease in elastin has also been documented [9, 17-19]. Electron microscopy findings revealed the presence of atypical collagen fibrils at the intra- and extracellular levels of the SMCs of the tunica media [20]. In contrast, some investigators have reported a reduction in the collagen content [21] with a concomitant decrease in elastin and an increase in total sugars [22], soluble nonscleroproteins [23] and glycosaminoglycans [24, 25]. More related work demonstrated that the elastin-to-collagen ratio was lower in the wall of VVs as compared to normal veins [26]. Contradictory evidence also exists on the pathology of SMCs in VVs. Several studies have reported an increase in the number or activity of SMCs [27-29] whereas other studies demonstrated a decrease in the number of SMCs associated with replacement by connective tissue elements [9]. Still other investigations failed to demonstrate any significant difference in SMC number and activity in the wall of VVs as compared to control [30].

In light of the contradicting results in relation to the nature of the abnormalities seen in the wall of VVs as far as the extracellular and cellular components are concerned, and since only few electron microscopy investigations have been conducted in relation to that matter, we decided to carry out a compressive study of the morphological changes taking place in VVs using saphenous veins and transmission electron microscopy. This study may shed some light on the events that take place in the course of varicosis and may pave the way to understand the structural and functional derangements occurring in the lower limb veins and to resolve existing disagreements.

\section{Material and Methods}

Subjects

VV samples were collected from 10 patients ( 4 males and $6 \mathrm{fe}-$ males) aged between 37 and 50 years (mean age 44 ). The patients were diagnosed as having primary varicosis and underwent stripping operations of the long saphenous vein. All patients had venous reflux as demonstrated clinically by continuous-wave Doppler probe examinations. Samples were taken from unstripped proximal varicose segments (near to the insertion into the common femoral vein) and from stripped ones (below the knee region). $\mathrm{Pa}-$ tients who had previously undergone sclerotherapy were excluded from the study. Control samples were collected from 10 age- and

Fig. 1. Electron micrographs of the tunica intima and tunica media of normal saphenous veins. A-C Tissue sections showing regular SMCs $(\mathrm{Sm})$ in cross-sectional $(\mathbf{A}, \times 3,300)$ and longitudinal $(\mathbf{B}$, $\times 5,000 ; \mathbf{C}, \times 2,100)$ arrangement. Note that the cells have a homogeneous sarcoplasm and few small intracytoplasmic vacuoles (arrows). Few vacuoles can also be seen closer to the sarcolemma in the sarcoplasm. Little extracellular space is seen among the SMCs, and it mainly contains small amounts of regularly arranged collagen fibers (arrowheads). Note the absence of elastic fibers in the extracellular space. E This section shows normal-appearing SMCs (Sm) in the subendothelial area of the vein. The subendothelial connective tissue contains normally arranged collagen fibers surrounding SMCs. Note also the normally appearing aggregates of elastic fi- 

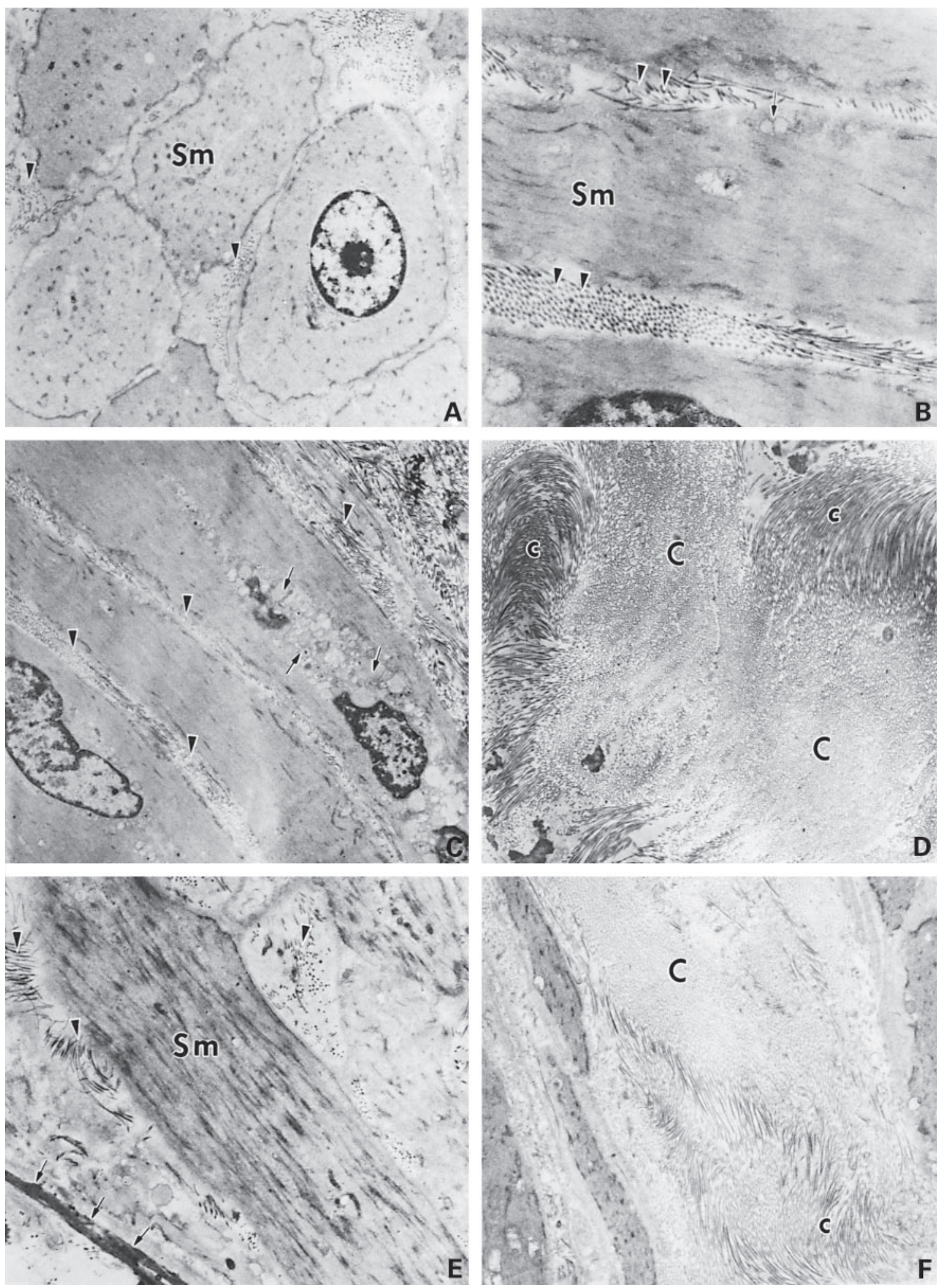

bers (arrows) at the junction of tunica intima and media representing the regular pattern of the internal elastic lamina. $\times 3,300$. D, $\mathbf{F}$ High-magnification view of the extracellular matrix in the tunica media $(\mathbf{D}, \times 2,500)$ and intima $(\mathbf{F}, \times 3,300)$ showing normally arranged collagen bundles in cross-sectional (upper-case C) and longitudinal (lower-case c) arrangement. The size and density of these collagen bundles are normal. Note the absence of elastic fibers among the collagen bundles. 
sex-matched patients with no history or clinical evidence of VVs and who underwent operative repair of vascular injuries. The study was conducted following the approval of the Human Ethics Committee at King Khalid Hospital (approval No. 64513), and a signed informed consent was obtained from each patient enrolled in the study.

\section{Preparation and Fixation of Tissue Samples}

Tissue samples were immediately fixed in freshly prepared 3\% glutaraldehyde and $10 \%$ formaldehyde in $0.1 \mathrm{M}$ sodium cacodylate buffer (pH 7.4). The samples were then cut into pieces of $1-2 \mathrm{~mm}^{3}$ and kept overnight at $4^{\circ} \mathrm{C}$ in the same fixative. Washing $(2 \times 30$ $\mathrm{min}$ ) was done the next day using $0.1 \mathrm{M}$ sodium cacodylate buffer, and postfixation for $1 \mathrm{~h}$ with $2 \%$ osmium tetraoxide followed. Next, the samples were dehydrated with ethyl alcohol and embedded in Spur's/Epon (6:4) resin [31, 32]. Sectioning $(2 \mu \mathrm{m})$ was performed using a Reichert-Jung Utrecht microtome, and staining followed using toluidine blue. These sections were used for light microscopy examination and for the determination of the appropriate areas of the vein to be selected for ultrathin sectioning. Fifteen ultrathin sections per case (silver-gold interference color) were placed on 300mesh nickel grids and were allowed to dry before being counterstained for 10-15 min with freshly prepared uranyl acetate. The ultrathin sections were then washed with distilled water (10 dips in 3 separate beakers) and stained for $10 \mathrm{~min}$ using lead citrate. The sections were finally rinsed with distilled water and dried [31, 32].

Examination of the ultrathin sections was performed using a Jeol 1200EX II transmission electron microscope at $80 \mathrm{kV}$. Fifteen micrographs were taken randomly of each section examined, and the components of the tunica intima and media of VVs and controls were analyzed using a double-blind method [31, 32].

\section{Results}

\section{Normal Control}

The electron microscopy evaluation of the wall of the veins taken from controls revealed that the SMCs in the tunica media were regularly distributed and showed no apparent hypertrophy or hyperplasia. The cells were surrounded by a fine layer of amorphous fibrous tissue and had well-marked nuclei with clear chromatin and prominent nucleoli (fig. 1A-C). The cytoplasm of the cells showed no abnormality. The tunica adventitia showed mainly collagen bundles which were oriented in various directions and which were separated by large gaps. Very few elastic fibers were present in this layer. In addition, collagen present underneath the endothelium was evenly distributed. A similar distribution was observed with the elastic fibers in both the tunica intima and media, where the fibers were localized mainly within well-defined internal and external elastic lamina (fig. 1E). The tunica intima showed a regular and uniform basal lamina of the endothelium along with SMCs having a characteristically abundant thin filamentous cytoplasm surrounded by thin external lamina (fig. 1C). Both the tunica intima and media showed a regular longitudinal and horizontal arrangement of collagen bundles (fig. 1F, D). The size and density of the collagen fibers were normal.

\section{Varicose Veins}

Electron microscopy observations of the tunica intima of VVs showed a significant proliferation and hypertrophy of the subendothelial SMCs associated with an increased subendothelial deposition of collagen and elastic fibers, features not observed in the normal control veins (fig. 2A-C). The SMC proliferation was distributed focally throughout the affected VVs forming focal patches that bulged into the vessel lumen. The hypertrophied SMCs that were widely invading the subintimal area showed a disrupted sarcolemma and occupied most of the subendothelial space of the tunica intima (fig. 2B, C). A higher-magnification view of these cells showed several large cytoplasmic vacuoles particularly in the perinuclear area, implying activated or modified SMCs. The vacuoles were of various sizes and shapes. Some vacuoles contained collagen-like material of varying electron density, while others resembled secondary lysosomes (fig. 2A-C). Some of the fields examined showed fragmented SMCs separated by increased amounts of extracellular matrix (fig. 3). This reflects a process of degradation and degeneration of SMCs associated with a replacement of such cells by connective tissue elements (fig. 2B, 3A, B). In fact, signs of degeneration and alterations in structure were also seen among these elements (fig. 2B). No proliferation of smooth or rough endoplasmic reticula was observed in the tissue sections examined. The internal elastic lamina appeared distorted with increased amounts of collagen fibers present inside the lamina (fig. 2A, B).

The VVs examined showed severely altered subendothelial areas associated with increased amounts of abnormally deposited collagen bundles and elastic fibers causing more invaginations in the lumen of the veins (fig. 3A, $\mathrm{C})$. The collagen fibers formed patches of woven, immature, broken and hydrolyzed collagen bundles. A similar abnormality was observed with the elastic fibers (fig. 4AC). An intensive disorganization and fragmentation of collagen bundles interwoven with abnormal elastic fibers were also prominent in the extracellular space in the tunica intima (fig. 4A, B). In the severely affected VVs, the intimal lining displayed a significant amount of fibrosis in which focal patches of fragmented SMCs were embedded. A significant increase in elastin fibers was observed (fig. 4C). 

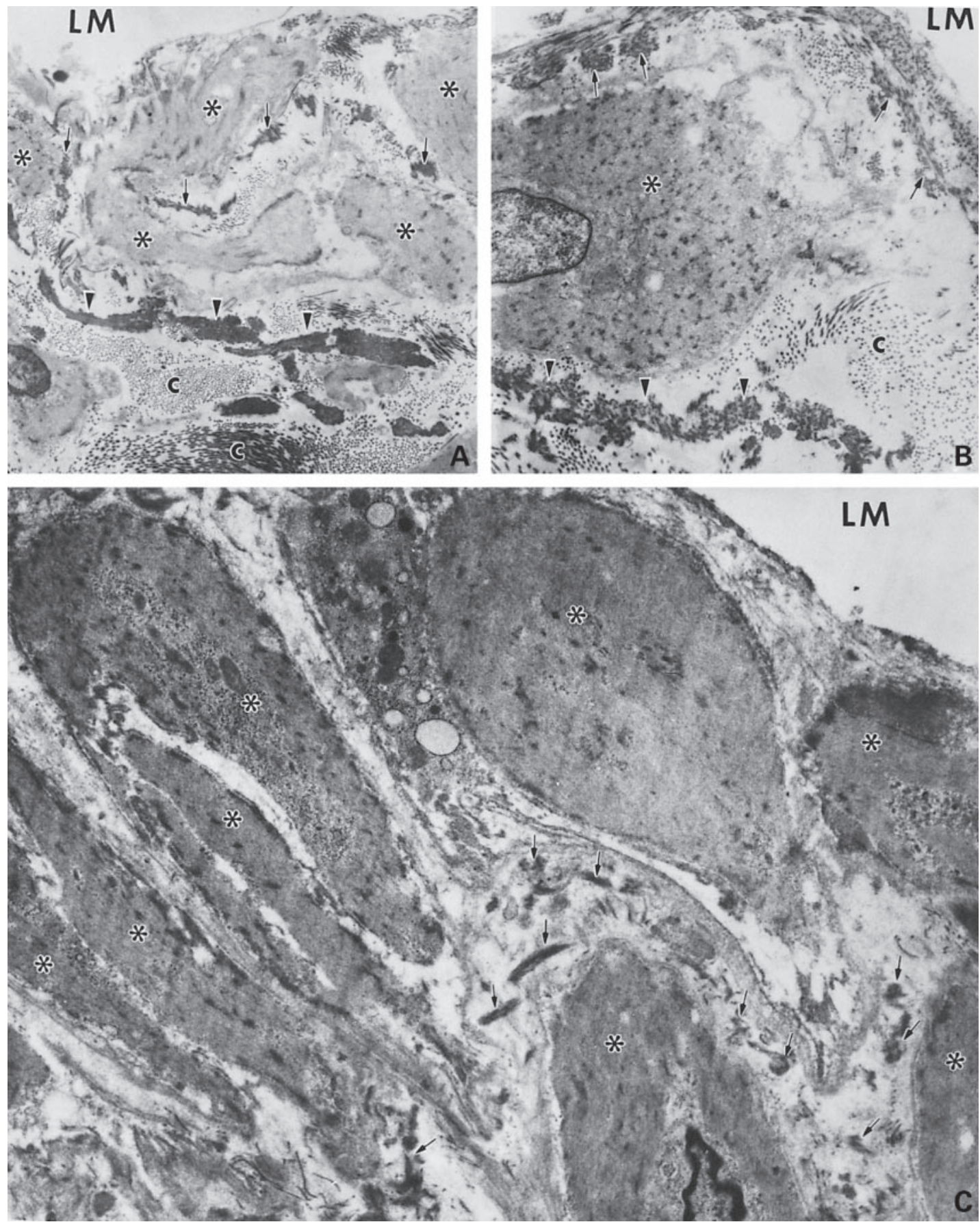

Fig. 2. Electron micrographs of the tunica intima of saphenous VVs. A A section showing an increased subendothelial deposition of collagen (c) and elastic (arrows) fibers. Note also the variable amount of abnormally arranged collagen fibers intermingled with elastic fibers (arrows). The internal elastic lamina (arrowheads) looks distorted with an increased amount of collagen fibers inside the lamina. The SMCs (asterisks) are widely seen invading the subintimal area. $\mathrm{LM}=$ Vessel lumen. $\times 3,100$. B An ultrathin tissue section showing a severely affected intimal lining of a VV. Note the significant amount of degenerated (arrowheads) fibrotic elastic fi- bers along with some disrupted collagen fibrils. The internal elastic lamina seems severely disrupted (arrowheads). Note the abnormal hypertrophied SMCs (asterisk) with disrupted sarcolemma. LM = Vessel lumen. $\times 4,700$. C A tissue section showing proliferation and hypertrophy of SMCs (asterisks). Note that the cells are swollen and occupy most of the subendothelial space in the intima. The SMCs seem to be replacing the extracellular matrix components of the subendothelial space. Fibrotic fragments of elastic fibers are seen scattered among the cells (arrows). $\mathrm{LM}=$ Vessel lumen. $\times 5,500$. 

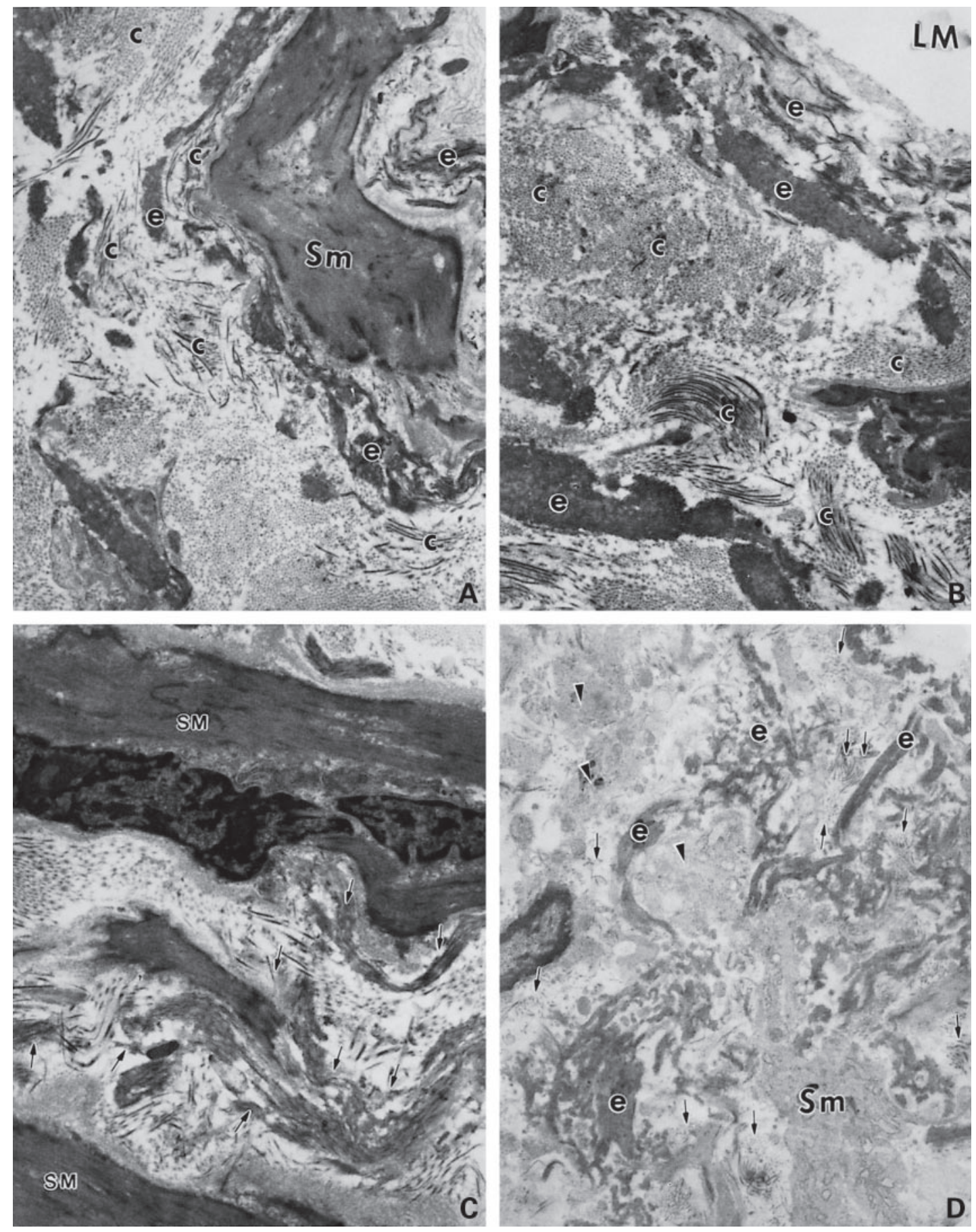

Fig. 3. Electron micrographs of severely affected tunica intima of saphenous VVs. A A tissue section showing a significant increase in the elastic fiber (e) deposition surrounding abnormal SMCs $(\mathrm{Sm})$. Note the increased amount of disorganized collagen fiber (c) deposition in the subintimal area. $\times 3,900$. B A VV section showing a severely affected subendothelial area. Note the increased amount of abnormally deposited collagen (c) bundles and elastic (e) deposits completely replacing the normal subendothelial area and bulging into the vessel lumen $(\mathrm{LM}) . \times 3,900$. C This section shows an area between two SMCs (SM) that is replaced by an increased amount of fragmented elastic fibers and collagen bundles (arrows). $\times 3,900$. D An ultrathin tissue section of a severely affected intimal lining of a VV. Note the significant amount of degenerated fibrotic elastic aggregations (e) along with disrupted collagen fibrils (arrows) scattered within the subendothelial area. Fragments (arrowheads) and extensions of SMCs $(\mathrm{Sm})$ are also seen embedded within these fibrotic areas of the affected intima. $\times 3,900$. 

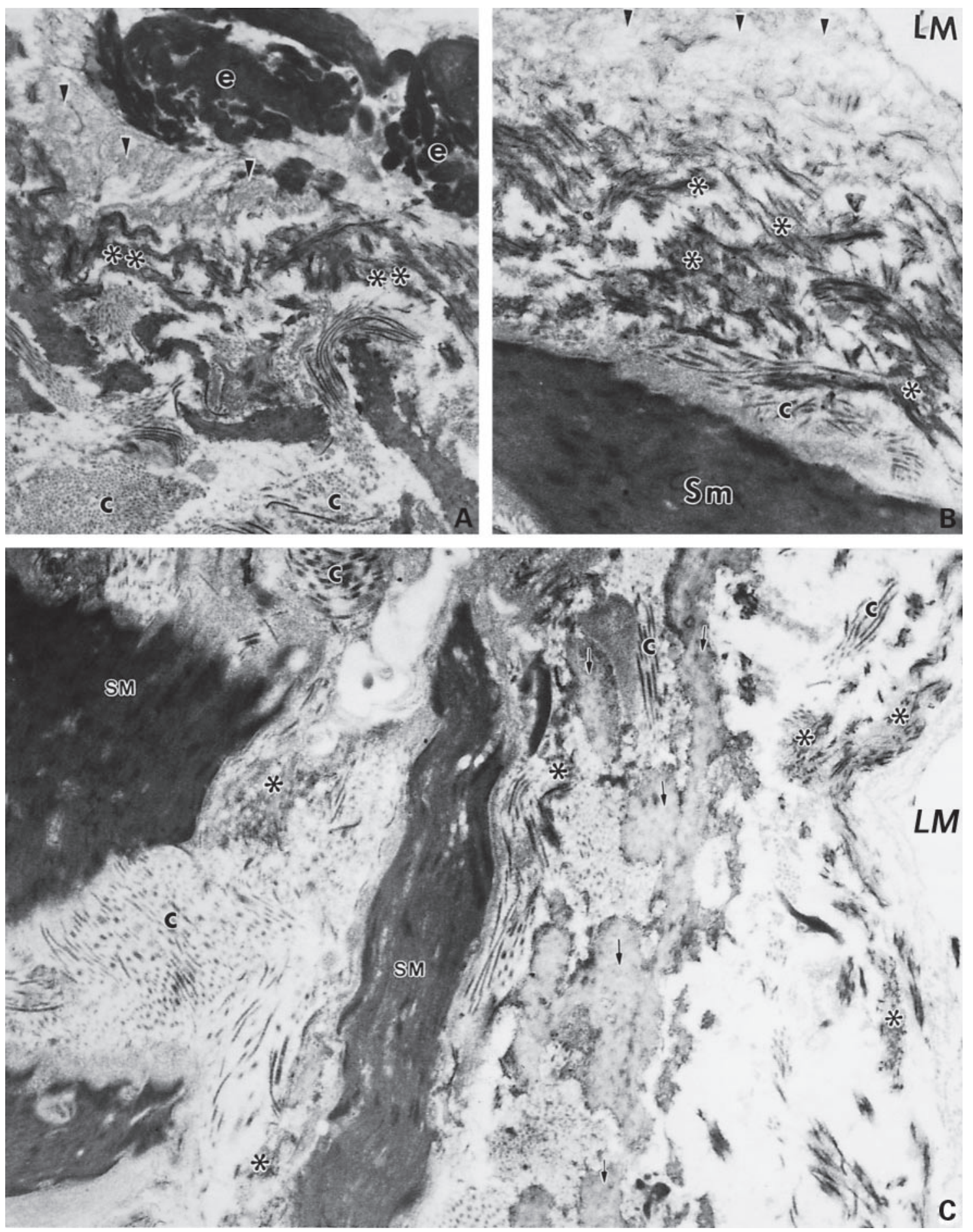

Fig. 4. Electron micrographs of the tunica intima of a severely affected saphenous VV. A, B A high-magnification view of the subendothelial area of the tunica intima showing an extensive amount of immature, hydrolyzed (arrowheads) and fragmented (asterisks) collagen fibers and bundles interwoven with abnormal elastic fibrils. Also, note the deposition of abnormally looking elastic aggregations (e) $\cdot \mathrm{LM}=$ Vessel lumen; $\mathrm{Sm}=$ smooth muscle cell. $\times 78,000$.
C A high-magnification view of the subintimal area showing an enormous increase in elastin fibers (asterisks) that are mixed with fragments of immature collagen fibrils (c). Note the large increase in fibrotic tissue (arrows) close to abnormally appearing SMCs (SM). The latter do not show a clear regular sarcolemma. Fragmented collagen bundles are very evident in the subendothelial region. $\times 94,000$. 
One of the striking changes observed in the tunica media of VVs was the separation of SMCs by the increased amount of extracellular matrix as compared to normal controls (fig. 5A, B). The regular arrangement of the extracellular matrix, particularly of collagen fibers, often seen in the normal vessel wall, was lost in the VV samples that we examined. The collagen fibers surrounding the SMCs of the tunica media were not grouped in their usual bundles but rather dispersed haphazardly in different directions. Some fibers were of small size and were found mostly isolated in groups of 2 or 3 (fig. 6A, B), whereas others were present in small vacuoles (fig. 6B). Areas of focal degeneration and patchy fibrosis were observed throughout the tunica media (fig. 6C, D). The focal degeneration of collagen fibers was extensive in some fields, while in others the degenerated collagen was completely replaced by homogeneous amorphous materials (fig. 6D).

Membrane-bound vesicles were widely scattered in the SMCs' cytoplasm of the tunica media which showed signs of hypertrophy (fig. 7A, B). Some of these vesicles were also segregated in the perinuclear area and contained collagen-like material of varied electron density, size and shape. Some nuclei of the SMCs showed condensed chromatin while others displayed a heterochromatic appearance (fig. 7B).

The elastic fibers in the extracellular matrix of the tunica media had a lighter density than that of collagen fibers. A significant abnormal increase in elastic fiber content was observed in areas of the media where there were degradations and degeneration of collagen fibers (fig. 8A-D). Elastin seems to be replacing the distorted collagen fibers and fragmented SMCs (fig. 8D). Abnormal accumulation of elastic bundles just adjacent to the SMCs' sarcolemma was also seen separating these cells (fig. 8B).

Another striking finding which was observed in the VV samples was the presence of 'ghost' bodies (fig. 9A, B). These bodies were large membrane-bound vesicles that were widely present in the extracellular matrix of the tunica media. They varied in size and shape, and contained various amounts of electron-dense substances the identity of which could not be determined at this stage of the study. The 'ghost' bodies were surrounded by fragmented collagen fibers. No significant changes were observed in the tunica adventitia of VVs.

\section{Discussion}

In our study, an important finding using transmission electron microscopy was the significant increase in the extracellular matrix of the tunica media of VVs as compared to normal control veins. Similar findings were reported by Sansilvestri-Morel et al. [33]. The SMCs were separated by an increased amount of fragmented and immature collagen as well as elastin tissue. Such an increase in the extracellular matrix components could either be a compensatory mechanism for the gradual loss of the SMCs' normal function (phenotype) following an external stimulus or could have preceded and led to such a loss. Regardless of which came first, our study demonstrated the presence of abnormal SMCs with heterochromatic nuclei and processes similar to the findings by BadierCommander et al. [34], who reported changes in SMC phenotype in VVs. Although the process that initiates changes in the phenotype of the SMCs in varicosis is still unclear, there seems to be a better understanding of the nature of the stimulus resulting in an increase in the extracellular matrix with a probable subsequent alteration in the SMC function. It has been argued that the presence of mast cells in the wall of VVs stimulates the proliferation of fibroblasts and the subsequent deposition of fibers through the release of basic fibroblast growth factor. Haviarova et al. [35] also suggested the important role of basic fibroblast growth factor in fibroproliferative disorders and associated its release with the development of medial fibrosis in VVs. A similar association was reported by Badier-Commander et al. [34]. Lengyel and Acsady [17] suggested that local hypoxia could directly lead to the production of certain bioactive substances, which in turn could result in the increased production of extracellular matrix followed by relaxation of SMCs. On the other hand, Lee et al. [36] argued that soluble vasoregulators associated with an abnormal increase in extracellular matrix modulate the basic tone and the response of SMCs by either modulating signal transduction or by altering the resting mechanical tension, which is transmitted across integrin and thus affects directly the isometric tension of SMCs. The authors reported that cytoskeleton stiffness and integrin/fibronectin density can modulate the contraction of the SMCs, since fibronectin, a multifunctional high-molecular-weight glycoprotein, is present in particular concentration around the SMCs where it forms filaments that attach at one end to the receptor on the muscle cell and to the collagen or elastic fibers at the other end [8]. 

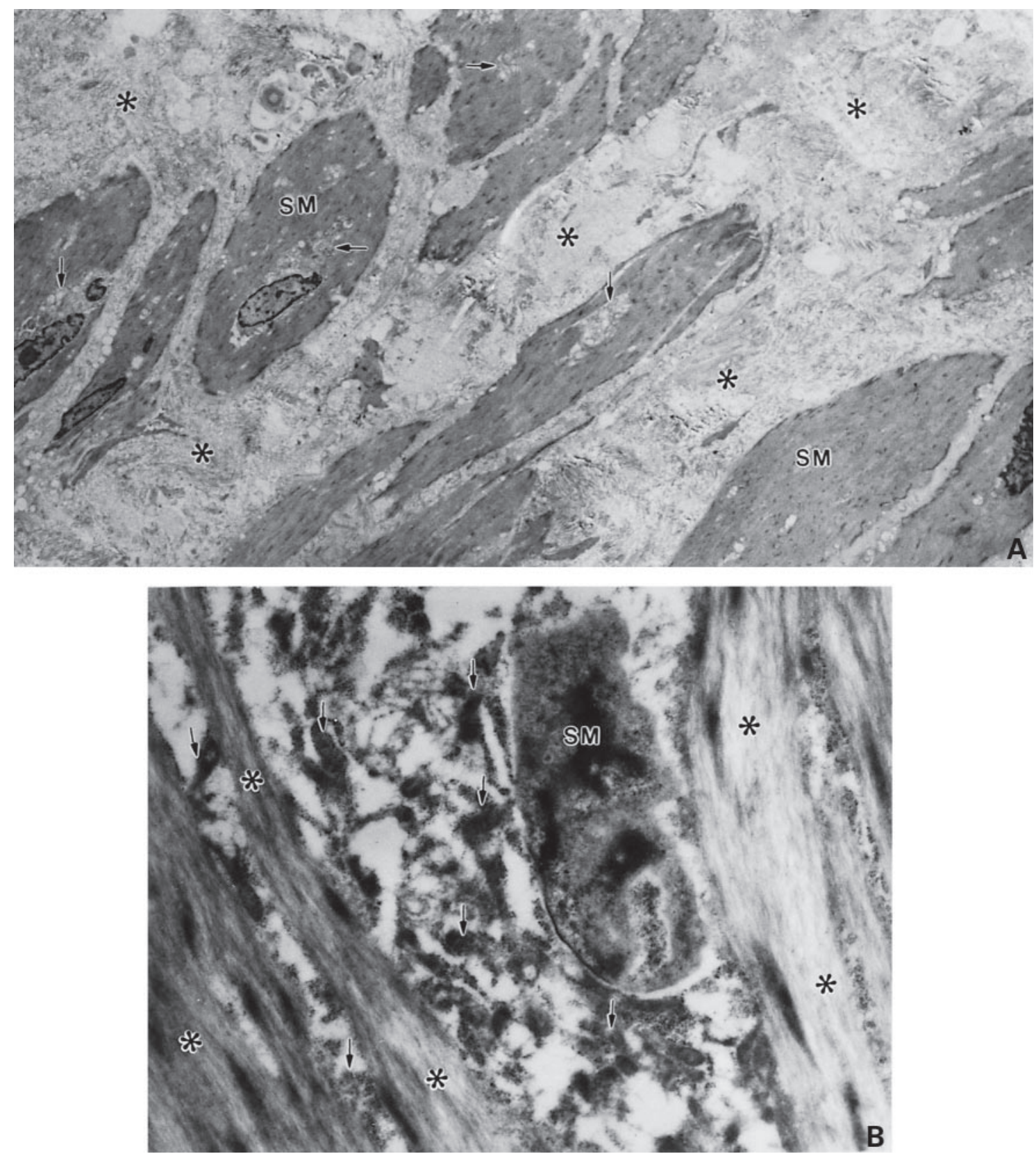

Fig. 5. Electron micrographs of the tunica media of a saphenous VV. A A tissue section of the tunica media of a VV showing an increased amount of collagen deposition among severely hypertrophied SMCs (SM). Note the separation of the cells by variable bands of abnormally arranged collagen fibers (asterisks). Note also that the SMCs contain an increased number of membrane-bound vesicles (arrows). $\times 1,500$. B A higher-magnification view showing severely disrupted SMCs (SM). Note that the cells show advanced degeneration and disintegration of their intracellular fibrils (asterisks). They are separated by an abnormal amount of disorganized elastic disposition (arrows) and collagen fragments. $\times 78,000$. 

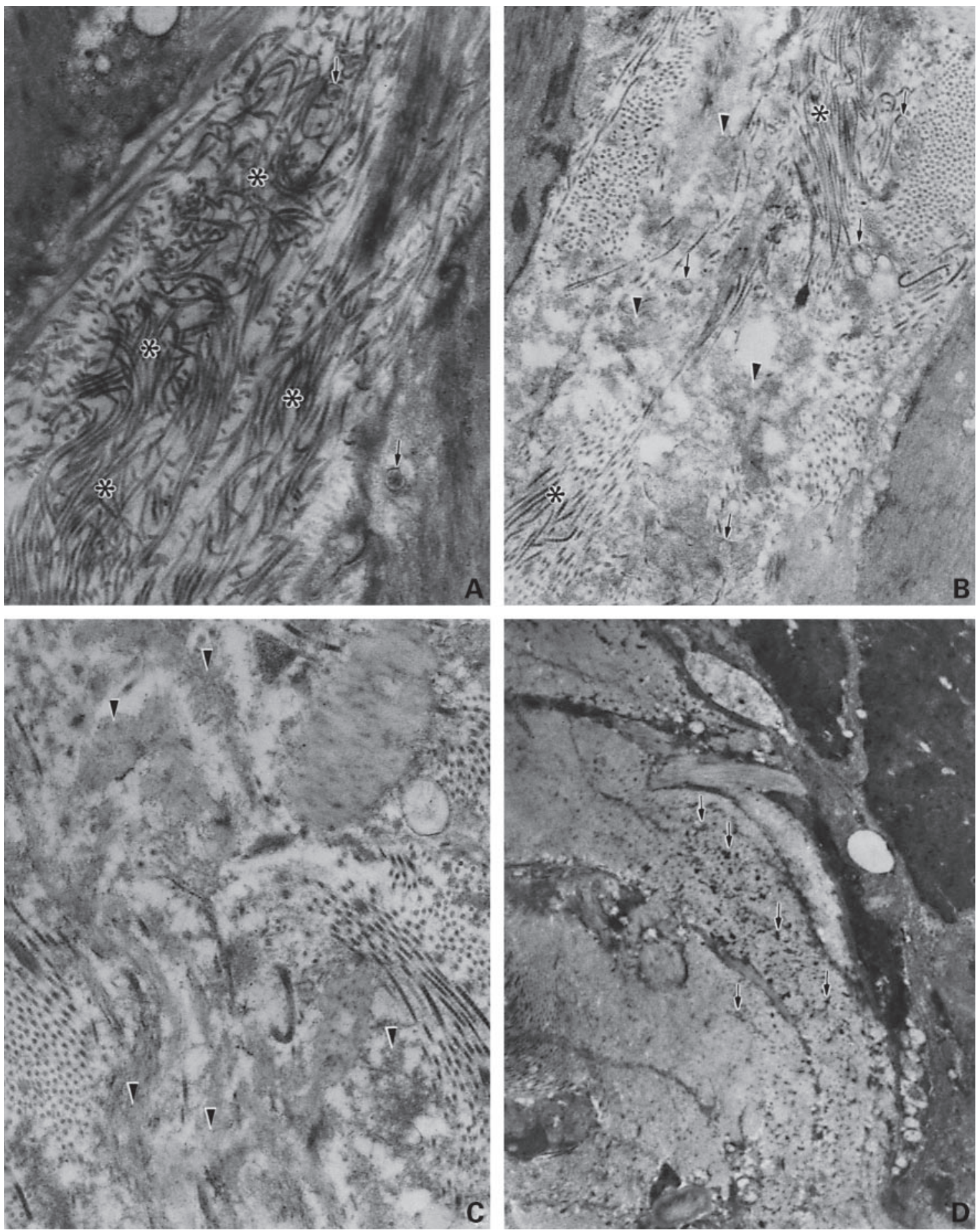

Fig. 6. Electron micrographs of the tunica media of saphenous VVs showing changes in the collagen fibers in the extracellular matrix. A-C These ultrathin sections show disorganized extracellular collagen fibers (asterisks). The fibers are of variable electron density and size, and are scattered in the extracellular matrix. They seem to be gradually replaced by fine granular material of moderate electron density suggesting focal disintegration of the extracellular sub- stance (arrowheads). Small vacuoles containing collagen (arrows) are also evident. A $\times 78,000$, B $\times 6,200, \mathbf{C} \times 78,000$. D A tissue section showing an advanced stage of collagen disintegration and changes in the extracellular matrix of the tunica media. The large areas of fibrotic changes seem to replace the fragmented and abnormally looking collagen fibrils (arrows). $\times 3,900$. 

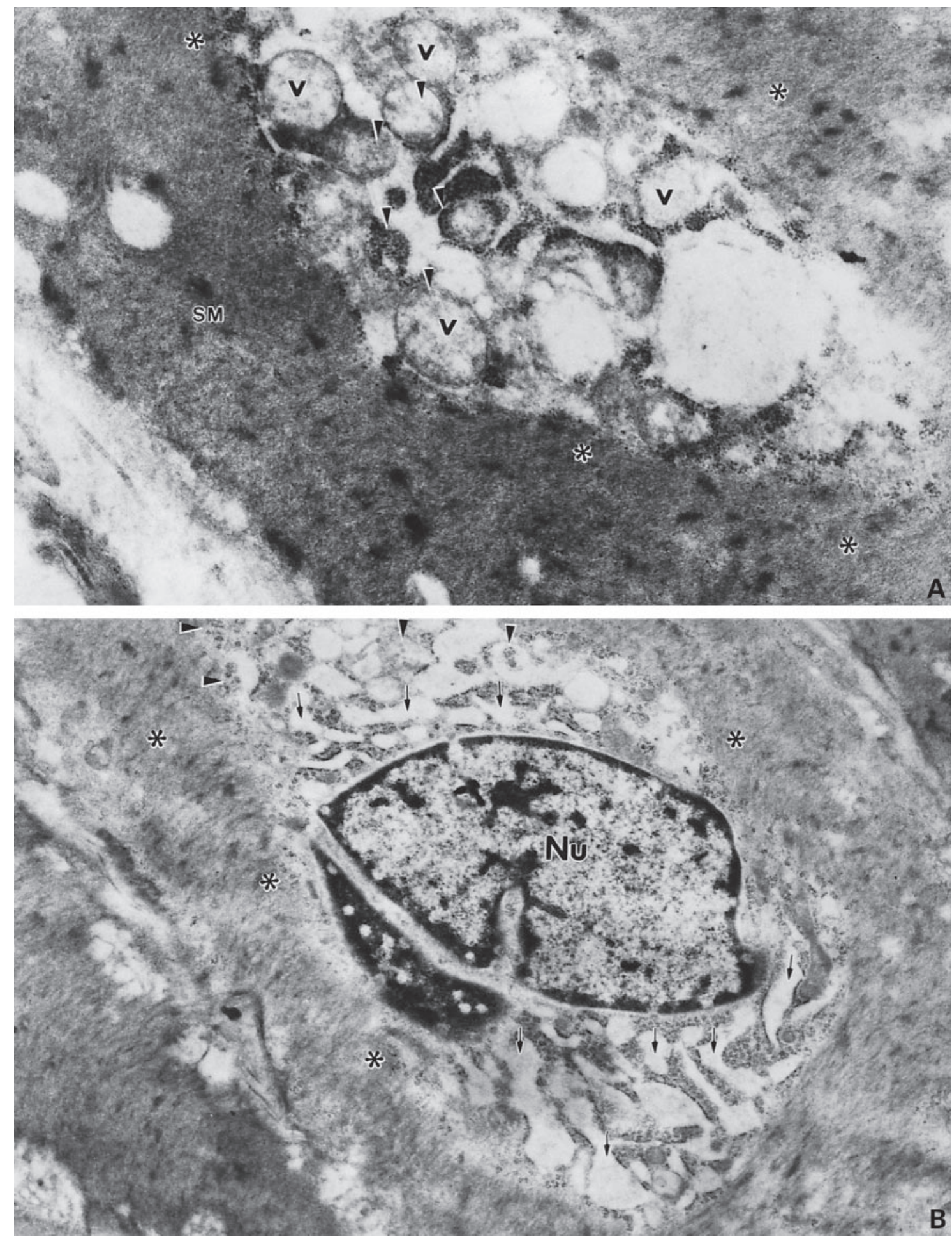

Fig. 7. Electron micrographs of the severely affected tunica media of a saphenous VV. SMCs (SM) of the tunica media showing variable membrane-bound vesicles (v; arrowheads). These vesicles appear to contain collagen-like material of variable density. The hypertrophied SMCs also show nonhomogeneous cytoplasm and ab- normally arranged intracellular fibrils (asterisks). Note the inactive SMC containing a heterochromatic nucleus $(\mathrm{Nu})$ with widely separated spaces seen in the perinuclear area (arrows). $\mathbf{A} \times 9,700$, B $\times 4,800$. 

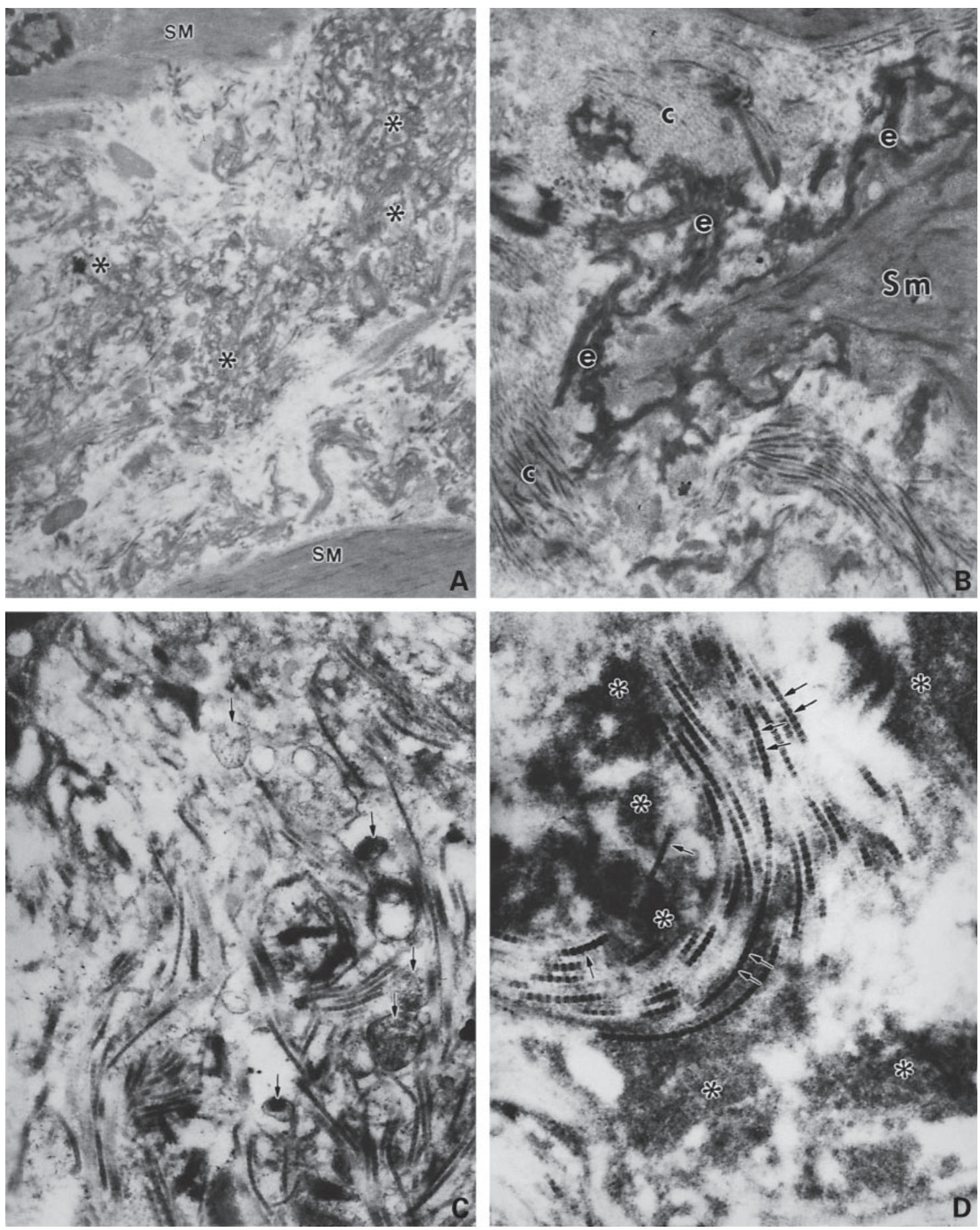

Fig. 8. Electron micrographs of the tunica media of saphenous VVs showing changes in the elastic fibers in the extracellular matrix. A Abnormal infiltration of elastic fibers intermingled with collagen fibrils (asterisks) is seen between two SMCs (SM) in this section. Note that the collagen bundles are severely disrupted and fragmented. $\times 3,900$. B This section shows abnormal accumulation of elastic bundles (e) just adjacent to SMC (Sm) sarcolemma. Patches of fragmented collagen aggregates (c) are also apparent in the extracellular matrix. $\times 6,300$. C A high-magnification view of an area with significant abnormal elastic tissue deposition. The damaged and disrupted elastic tissue fills the extracellular matrix and mingles among the fragmented, distorted and disorganized collagen fibrils. Note the presence of ghost bodies that seem to contain immature and hydrolyzed collagen fibers (arrows). $\times 78,000$. D A high-magnification view showing fragmented collagen bundles (arrows) being replaced by a homogeneous amorphous material that appears to be necrotic patches (asterisks). $\times 15,000$. 

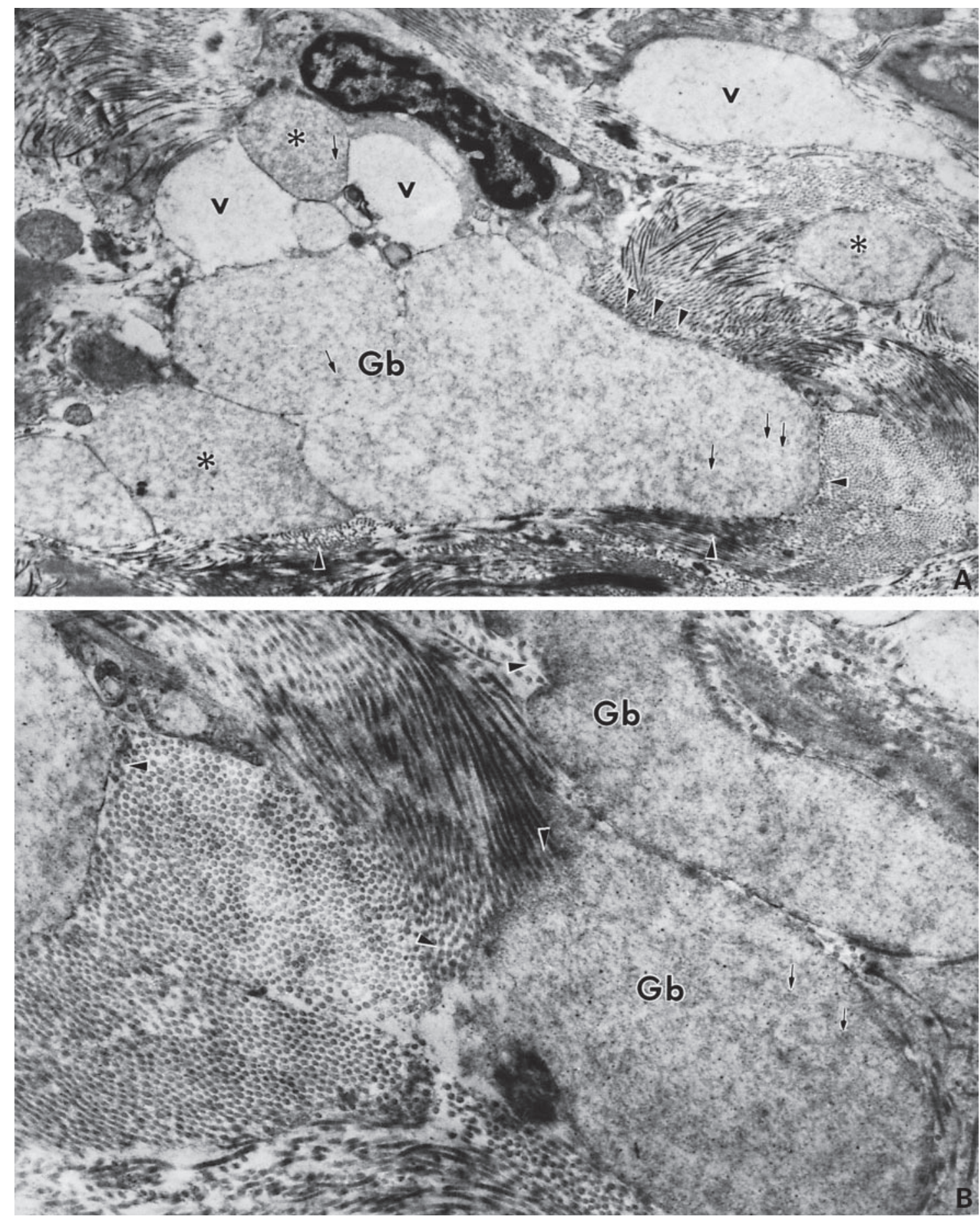

Fig. 9. Electron micrographs of the tunica media of saphenous VVs showing ghost bodies in the extracellular matrix. Tissue sections show accumulation of large ghost bodies $(\mathrm{Gb})$ in the extracellular matrix. These bodies are membrane-bound vesicles of different sizes and shapes (asterisks) with varying amounts of an electrondense substance. The latter appears to be degenerated and imma- ture collagen fibrils (arrows). Note that some vacuoles are scattered among abnormally fragmented collagen fibers and in close association with the ghost bodies. Also note the intimate contact of the ghost bodies with the surrounding collagen fibrils (arrowheads) suggesting that these bodies may be involved in engulfing the collagen fibrils. A $\times 3,100, \mathbf{B} \times 78,000$. 
The results of the present study demonstrated a significant increase in elastic fibers in the intima and media of VVs at the electron microscopy level. Similar findings were reported by Travers et al. [3] where an increase in the media but not intimal elastic fiber content was observed. Our results and those of Travers et al. contradict the findings by Venturi et al. [26], who suggested that varicosis develops through different stages. In an early stage, more synthesis or reduced catabolism of collagen could partially compensate for the loss of elastin without replacing the primary structure of the vein. In a later stage, the vein becomes dilated with a significant decrease in both collagen and elastin and subsequent loss of elasticity and strength [26]. This confirms previous reports where the elastic fiber content of the wall of VVs was found to decrease as the disease develops $[11,26]$. One might argue that an increase in the elastic fiber content of the wall of VVs should provide the veins with a better recoil ability, thus putting less pressure on the vessel wall. However, the nature and function of elastic fibers seen in VVs could be different from what is usually observed in normal veins. Whether such an argument is tenable remains to be seen.

In our study, the presence of 'ghost' bodies in the media of VVs and their absence in normal veins may suggest a possible initial effort exercised by the SMCs to limit the invasion by the extracellular matrix in general and collagen in particular. Such bodies were also observed by Jurukova and Milenkov [37] in the tunica intima of saphenous VVs. The authors suggested that collagen ingestion and intracellular decomposition by SMCs could represent one of the specific mechanisms responsible for the remodeling of the blood vessel wall. Other mechanisms involve an elevation in the ratio of collagen type I to collagen types III and IV [33, 38] and an increase in the tissue inhibitor of metalloproteinase TIMP-1 [39, 40].

As far as the changes occurring in the tunica intima of VVs are concerned, the results of the present study clearly demonstrate intimal hypertrophy and hyperplasia of the subendothelial SMCs in all the samples analyzed. These findings are in agreement with previous observations $[3,37,41]$ but do not concur with the results obtained by Mashiah et al. [42], who did not observe any changes in the tunica intima of saphenous VVs using scanning electron microscopy. Whether the subendothelial and medial SMCs of VVs share the same trigger leading to the disruption of their normal function is still under investigation by our group. Nevertheless, it was interesting to notice that the changes occurring in the subendothelial SMCs did not correlate with any changes in the endothelial cells of the VVs studied. One possible expla- nation is that the latter do not go through the same cascade of events that the former undergo despite the fact that endothelial cells not only act as barriers but also as regulators of vascular tones [43]. Nevertheless, it has been reported that a significant increase in endothelial adhesion molecule ICAM-1 associated with more thromboxane $\mathrm{A}_{2}$ and prostaglandin $\mathrm{E}_{2}$ synthesis and with an increased interaction among leukocytes, platelets and endothelial cells is observed in VVs $[44,45]$. This could imply that although the morphology of endothelial cells may not change in varicosis the overall function of such cells may.

The data obtained from the present study in relation to the changes occurring in the intima of VVs support the findings by Jurukova and Milenkov [37] where vacuoles of various electron densities were found inside the subendothelial SMCs, mostly in the perinuclear area. The presence of such vacuoles may suggest a phagocytic role of SMCs as the disease develops. In fact, the observed hypertrophied rough endoplasmic reticula and Golgi apparatus of the collagen-containing SMCs in our study might reflect an accelerated synthesis of hydrolytic enzymes and primary lysosomes necessary for the completion of the degradation process of the collagen fibers. This could also explain the presence of abnormal collagen fibers in the vicinity of the SMCs.

Finally, in order to come up with a proper management of varicosis, it is essential to understand the basic mechanisms leading to the development of such a disease in people at various ages. Accordingly, a holistic approach should be taken when studying the various parameters involved in the disease, namely the cellular and extracellular components of the vessel wall. The basic current argument in varicosis revolves around the trigger(s) as well as the cascade of events leading to the formation of VVs. Therefore, a proper understanding of the nature of the trigger(s) and the step-by-step gradual changes occurring in the vessel wall of VVs may lead to not only a better treatment, but also prevention of the disease.

\section{Conclusion}

Findings in this study shed more light on the structural deformation taking place during varicosis and may help the physicians and surgeons develop better approaches to preventing as well as treating VVs.

\section{Acknowledgement}

The authors would like to thank Amal Wagdi, Mohammed J. Al-Galaaf and Refat Eid for their technical assistance. 


\section{References}

1 Bradbury A, Evans C, Allan P, Lee A, Ruckley CV, Fowkes FG: What are the symptoms of varicose veins? Edinburgh vein study cross sectional population survey. Br Med J 1999;318: 353-356.

2 Callam MJ: Epidemiology of varicose veins. B J Surg 1994;81:167-173.

-3 Travers JP, Brookes CE, Evans J, Baker DM, Kent C, Makin GS, Mayhew TM: Assessment of wall structure and composition of varicose veins with reference to collagen, elastin and smooth muscle content. Eur J Vasc Endovasc Surg 1996;11:230-237.

4 Bergan JJ: Treatment of superficial venous insufficiency of the legs. West J Med 1993;159: 686.

5 Tuchsen F, Krause N, Hannerz H, Burr H, Kristensen TS: Standing at work and varicose veins. Scand J Work Env Health 2000;26:414 420.

-6 Charles AK, Gresham GA: Histopathological changes in venous grafts and in varicose and non-varicose veins. J Clin Pathol 1993;46: 603-606.

7 Michiels C, Bouaziz N, Remacle J: Role of the endothelium and blood stasis in the development of varicose veins. Int Angiol 2002;21: $18-25$.

8 Michiels C, Bouaziz N, Remacle J: Role of the endothelium and blood stasis in the appearance of varicose veins. Int Angiol 2002;21:18.

-9 Rose SS, Ahmed A: Some thoughts on the aetiology of varicose veins. J Cardiovasc Surg 1986;27:534-543

10 Alexander CJ: The theoretical basis of varicose vein formation. Med J Austral 1972;1:258 261.

11 Clarke GH, Vasdekis SN, Hobbs JT, Nicolaides AN: Venous wall function in the pathogenesis of varicose veins. Surgery 1992;111: 402-408.

12 Leu HJ: Morphological aspects of changes in the venous wall. Phlebologie 1980;33:223231.

13 Leu HJ, Vogt M, Pfrunder H: Morphological alterations of non-varicose and varicose veins: a morphological contribution to the discussion on pathogenesis of varicose veins. Basic Res Cardiol 1979;74:435-444.

$\checkmark 14$ Golledge J, Quigley FG: Pathogenesis of varicose veins. Eur J Vasc Endovasc Surg 2003;25: 319-324.

15 Michiels C, Arnould T, Thibaut-Vercruyssen R, Bouaziz N, Janssens D, Remacle J: Perfused human saphenous veins for the study of the origin of varicose veins: role of the endothelium and of hypoxia. Int Angiol 1997;16:134141.

16 Bouissou H, Julian M, Pieraggi MT, Louge L: Essential varices of the internal saphenous vein. Bull Acad Nat Med Paris 1988;172:529_ 534.
17 Lengyel I, Acsady G: Histomorphological and pathobiochemical changes of varicose veins: a possible explanation of the development of varicosis. Acta Morphol Hung 1990;38:259267.

18 Maurel E, Azema C, Deloly J, Bouissou H: Collagen of the normal and the varicose human saphenous vein: a biochemical study. Clin Chim Acta 1990;193:27-37.

19 Prerovsky I, Kruszewska E, Linhart J, Hlavova A: Distensibility of the forearm veins in patients with primary varicose veins. Angiologica 1969;6:354-361.

20 Staubesand J: Intracellular collagen in smooth muscle: the fine structure of the artificially occluded rat artery and ureter, and of human varicose and arteriosclerotic vessels. Beitr Pathol 1977;161:187-193.

21 Psaila JV, Melhuish J: Viscoelastic properties and collagen content of the long saphenous vein in normal and varicose veins. Br J Surg 1989;76:37-40.

22 Svejcar J, Perovsky I, Linhart J, Kruml L: Content of collagen, elastin, and hexosamine in primary varicose veins. Clin Sci 1963;26:325330.

23 Andreotti L, Cammelli D, Banchi G, Guarnieri $\mathrm{M}$, Serantoni C: Collagen, elastin and sugar content in primary varicose veins. Ricerca Clin Lab 1978;8:273-285.

24 Drubaix I, Maraval M, Robert L, Robert AM: Hyaluronic acid (hyaluronan) levels in pathological human saphenous veins: effects of procyanidol oligomers. Pathol Biol (Paris) 1997; 45:86-91.

25 Drubaix I, Viljanen-Tarifa E, Robert AM, Robert L: Role of glycosoaminoglycans in venous disease: mode of action of some flavonoid drugs. Pathol Biol (Paris) 1995;43:467-470.

26 Venturi M, Bonavina L, Annoni F, Colombo L, Butera C, Peracchia A, Mussini E: Biochemical assay of collagen and elastin in the normal and varicose vein wall. J Surg Res 1996;60: 245-248.

-27 Corcos L, De Anna D, Dini M, Macchi C, Ferrari PA, Dini S: Proximal long saphenous vein valves in primary venous insufficiency. $\mathrm{J}$ Mal Vasc 2000;25:27-36.

-28 Svejcar J, Perovsky I, Linhart J, Kruml L: Content of collagen, elastin, and water in walls of the internal saphenous vein in man. Circ Res 1962;11:296-300.

29 Browse NL, Burnand KG, Thomas ML: Pathology, diagnosis, and treatment of vein diseases; in Arnold E (ed): Diseases of the Veins. London, Wiley, 1988, vol 2, pp 20-25.

30 Lees TA, Mantle D, Lambert D: Analysis of the smooth muscle content of the normal and varicose veins. Phleobology 1992;92:56-58.

31 Renno WM, Mahmoud MS, Hamdi A, Beitz AJ: Quantitative immunoelectron microscopic colocalization of GABA and enkephalin in the ventrocaudal periaqueductal gray of the rat. Synapse 1999;31:216-228.
32 Renno WM, Lee JH, Beitz AJ: Light and electron microscopic immunohistochemical localization of $\mathrm{N}$-acetylaspartylglutamate (NAAG) in the olivocerebellar pathway of the rat. Synapse 1997;26:140-154.

33 Sansilvestri-Morel P, Rupin A, Badier-Commander C, Kern P, Fabiani JN, Verbeuren TJ, Vanhoutte PM: Imbalance in the synthesis of collagen type I and collagen type III in smooth muscle cells derived from human varicose veins. J Vasc Res 2001;38:560-568.

-34 Badier-Commander C, Couvelard A, Henin D, Verbeuren T, Michel JB, Jacob MP: Smooth muscle cell modulation and cytokine overproduction in varicose veins: an in situ study. $\mathbf{J}$ Pathol 2001;193:398-407.

- 35 Haviarova Z, Weismann P, Pavlikova D, Durdik S, Kovac P, Stvrtinova V, Mraz P: Mast cell infiltration in the wall of varicose veins. Acta Histochem 2002;104:357-360.

36 Lee K, Tsai KY, Wang N, Ingber DE: Extracellular matrix and pulmonary hypertension: control of vascular smooth muscle cell contractility. Am J Physiol 1998;274:H76-H82.

- 37 Jurukova Z, Milenkov C: Ultrastructural evidence for collagen degradation in the walls of varicose veins. Exp Mol Pathol 1982;37:3747.

38 Waksman Y, Mashiah A, Hod I, Rose SS, Friedman A: Collagen subtype pattern in nor$\mathrm{mal}$ and varicose saphenous veins in humans. Isr J Med Sci 1997;33:81-86.

- 39 Woodside KJ, Hu M, Burke A, Murakami M, Pounds LL, Killewich LA, Daller JA, Hunter GC: Morphologic characteristics of varicose veins: possible role of metalloproteinases. J Vasc Surg 2003;38:162-169.

-40 Badier-Commander C, Verbeuren T, Lebard C, Michel JB, Jacob MP: Increased TIMP/ MMP ratio in varicose veins: a possible explanation for extracellular matrix accumulation. J Pathol 2000;192:105-112.

-41 Porto LC, Ferreira MA, Costa AM, da Silveira PR: Immunolabeling of type IV collagen, laminin, and alpha-smooth muscle actin cells in the intima of normal and varicose saphenous veins. Angiology 1998;49:391-398.

42 Mashiah A, Rose SS, Hod I: The scanning electron microscope in the pathology of varicose veins. Isr J Med Sci 1991;27:202-206.

43 Bassenge E: Endothelial function in different organs. Prog Cardiovasc Dis 1996;39:209228.

44 Takase S, Bergan JJ, Schmid-Schonbein G: Expression of adhesion molecules and cytokines on saphenous veins in chronic venous insufficiency. Ann Vasc Surg 2000;14:427-435.

45 Shebuski RJ, Kilgore KS: Role of inflammatory mediators in thrombogenesis. J Pharmacol Exp Ther 2002;300:729-735. 